\title{
Ultrastructure of Collagen Fibers and Distribution of Extracellular Matrix in the Temporomandibular Disk of the Human Fetus and Adult
}

\author{
By \\ Hiroshi TAKAHASHI and Iwao SATO \\ Department of Anatomy, School of Dentistry at Tokyo, The Nippon Dental University, Tokyo 102-8159 Japan \\ - Received for Publication, September 6, 2001 - \\ Key Words: Collagen fiber, Extracellular matrix, TMJ, Disk, Ultrastructure

\begin{abstract}
Summary: We quantitatively examined the distribution of these differences in extracellular matrices (collagen types I, III, and fibronectin) and elastic fibers under confocal laser scanning microscopy and electron scanning microscopy in terms of their contribution to the mechanics of the TMJ during development and in adults. Elastic fibers were found in the anterior and posterior bands in adults aged 40 years, and a few elastic fibers in the anterior band of the disk in adults aged 80 to 90 years. The extracellular matrix contents of the TMJ disk are shown in various detected levels in the anterior, intermediate, posterior bands of TMJ disk. During development, collagen fibers are arranged in a complex fashion from 28 weeks' gestation. These ultrastructures of the embryonic TMJ are resembled to that of adults aged the $40 \mathrm{~s}$, however the difference in extracellular matrix distribution found in embryonic stages and adults. They might reflect the differences in function between mastication and sucking or the changes in shape and form as results of functional disorders of the TMJ.
\end{abstract}

Biophysical stress on the temporomandibular joint (TMJ) disk is caused by continuous laxity followed by tension in jaw movement, and is reflected in the structural properties of the disk ( $\AA$ kerman et al., 1986; Isberg and Isacsson, 1986; Stratmann et al., 1996). During jaw movement the disk changes shape and becomes markedly thin and uneven (Öberg et al., 1971). Perforation of the central, dense part of the disk is often found in humans ( $\AA$ kerman et al., 1986). Under the stress of retrusive guidance of the mandible, the collagenous fibers of the disk become thin at the apex and anteriorly of the disk (Jonsson et al., 1999). Rees (1954) reported that the disk of the TMJ was composed of 4 regions; anterior band, intermediate band, posterior band, and bilaminar zone. Previous investigators have reported that the distribution of fibrous elastic fibers in the disk changes with age (Minarelli and Liberti, 1997), reflecting their functional role in jaw movement (Griffin and Sharpe, 1962; Nagy and Daniel, 1991; Ali et al., 1993; Mills et al., 1994). Extracellular matrix components are also found in the mammalian disk, where the mechanical stress of mastication is loaded (Nagy and Daniel, 1991; Ali et al., 1993; Isberg and Isacsson, 1986; Mills et al., 1994). The distribution of these components characterizes the loading stresses during jaw movement. We studied the distribution of the extracellular matrix components of the disk and the morphology of the collagenous fibers in terms of their contribution to the mechanics of the TMJ during development. Our aim was to answer the following questions: How does the human TMJ disk change form during development and aging? What roles do the different morphological features play in the development of jaw movement? How are extracellular matrix components distributed during development of the human TMJ?

We quantitatively examined the distribution of extracellular matrix components (collagen types I, III, and fibronectin) and elastic fibers under confocal laser scanning microscopy and electron scanning microscopy in 24 fetuses and in 54 adults.

\section{Materials and Methods}

Specimens

We examined 54 adults Japanese cadavers ( 32

Correspondence: Dr. Iwao Sato PhD., Department of Anatomy, School of Dentistry at Tokyo, The Nippon Dental University. 1-9-20 Fujimi, Chiyoda-ku, Tokyo 102-8159, Japan 
male and 22 female adults; 40 to 90 years of age) and 24 fetuses $(12,16,20,24,28,32$ weeks gestation). The fetuses were obtained from legally approved induced abortions and from spontaneous abortions. The approximate age of each fetus was assigned by reference to Streeter's Tables (Streeter, $1920,1942,1948,1951)$.

\section{Scanning Electron Microscopic Study}

Specimens (16 adults and 12 fetuses) from the TMJs processed at autopsy were fixed in $2 \%$ glutaraldehyde and postfixed in $1 \%$ osmic acid. They were washed in $0.1 \mathrm{M}$ cacodylate buffer ( $\mathrm{pH} 7.2$ ), etched with $8 \mathrm{~N}$ hydrogen chloride for $1 \mathrm{hr}$ at room temperature, and finally dried by a t-butyl alcohol freeze-drying. They were coated with a $1.5-\mathrm{nm}$ thick layer of gold palladium and observed under a scanning electron microscope (5 kv, S-4000, Hitachi Co., Tokyo, Japan).

\section{Light Microscopic Study}

Other specimens (38 adults and 12 fetuses) were fixed for a further $30 \mathrm{~min}$ in a solution of $10 \%$ formalin. After demineralization with ethylenediaminetetraacetic acid $(0.3 \mathrm{ml} / \mathrm{L})$ overnight at $4^{\circ} \mathrm{C}$ and washing in running water, the specimens were dehydrated in absolute ethyl alcohol and then embedded in paraffin. Serial sagittal and longitudinal sections were cut at a thickness of about $10 \mu \mathrm{m}$ with a rotary microtome. Sections were used for Elastic van Gieson staining and immunofluorescence staining.

\section{Immunofluorescence Antibodies}

The primary antibodies used were rabbit monoclonal antibodies directed against rat-tail tendon collagen type I (LB-1102; LSL Co., Tokyo, Japan) and bovine placental collagen III (LB-1387; LSL Co.), and bovine blood plasma fibronectin (LB1027, LSL Co.). The secondary antibodies used were fluorophore-labeled goat antibodies directed against rabbit or mouse IgG (Oregon Green antirabbit or anti-mouse IgG 514 goat anti-rabbit or anti-mouse IgG $(\mathrm{H}+\mathrm{L})$ conjugate, O-6383 or O-6384, Molecular Probes, Oregon, USA).

\section{Immunofluorescence Study}

Sections were treated with $0.05 \%$ trypsin for 15 min, incubated at $37^{\circ} \mathrm{C}$ with $0.01 \mathrm{M}$ Tris-buffered

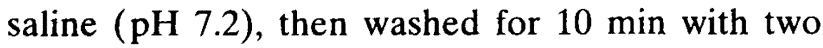
changes of phosphate-buffered saline (PBS, pH 7.2). After separate incubations with the primary antibodies against fibronectin and collagen types I and III, the sections were washed with PBS and, after a second incubation with the secondary antibodies for $1 \mathrm{~h}$ at room temperature, the specimens and controls (treated with normal rabbit serum in PBS) were washed with PBS. The sections were then mounted with $90 \%$ glycerin and examined under a Zeiss photomicroscope equipped with epifluorescence optics (TCS SP, $488 \mathrm{~nm}$; Leika; Wetzlar, Germany) and by confocal laser scanning microscopy (CLSM, Leika; Wetzlar, Germany).

\section{Results}

\section{Elastic van Gieson Staining}

In the fetuses, elastic fibers were not found in the TMJ disk. In contrast, in adults in the 40s, elastic fibers were found in the anterior and posterior bands with regular and loose elastic fibers parallel to the collagen bundles of the disk (Fig. 1a, b). There were only a few elastic fibers in the anterior band of the disk in adults aged 80 to 90 years (Fig. 1c, d).

\section{Immunofluorescence Observation}

The extracellular matrix (collagen types I and III and fibronectin) contents of the TMJ disk are shown in figures 2-4. The concentration of collagen type I (CoI) in the disk increased from 24 weeks' gestation, and at 32 weeks' gestation CoI was detected mainly in the intermediate band of the disk. Collagen type III (CoIII) was detected in the disk at 32 weeks' gestation; its concentration increased in the intermediate band from 24 weeks' gestation (Figs. 2-3, Table 1). In adults, the concentration of the CoIII in the intermediate and posterior bands was higher than in the anterior band (Fig. 4, Table 1). In fetuses at 12 and 16 weeks' gestation, the concentration of fibronectin (FN) was still low in the posterior band of the disk (Figs. 2-3, Table 1). The concentration increased from 20 weeks' gestation to 32 weeks' gestation. And then the $\mathrm{FN}$ was also high in examined adult age groups (Fig. 4, Table 1).

\section{Scanning Electron Microscopic Observation}

At 12 weeks' gestation, collagen fibers formed thin layers in the disk. The disk was composed of a few of these collagen layers, which had a networklike structure. From 16 weeks' gestation, the disk had thick anterior and posterior bands and a thin intermediate band. In the intermediate band of the disk, the collagen fibers formed bundles oriented anteroposteriorly. Transversely oriented fibers were found in the inner layer of the disk. From 20 weeks' gestation, the disk had a dense compound layer structure with a complex arrangement of fibers. Many transversely oriented collagen bundles with loop-like structures lay between anterolaterally 
Table 1. Distribution and intensity of immunostaining for collagenous and non-collagenous protein in the TMJ 12, $16,20,24,28,32$ weeks gestation and adult ages of $80 \mathrm{~s}-90 \mathrm{~s}$

\begin{tabular}{|c|c|c|c|c|}
\hline Weeks & Antibody & Ant. & Int. & Post. \\
\hline \multirow{3}{*}{12} & CoI & - & - & - \\
\hline & CoIII & - & - & - \\
\hline & $\mathrm{FN}$ & - & - & \pm \\
\hline \multirow{3}{*}{16} & $\mathrm{Col}$ & - & - & \pm \\
\hline & CoIII & \pm & \pm & \pm \\
\hline & FN & - & - & \pm \\
\hline \multirow{3}{*}{20} & CoI & - & - & \pm \\
\hline & CoIII & \pm & \pm & \pm \\
\hline & FN & \pm & \pm & \pm \\
\hline \multirow{3}{*}{24} & CoI & + & + & + \\
\hline & ColIII & \pm & + & \pm \\
\hline & $\mathrm{FN}$ & \pm & \pm & \pm \\
\hline \multirow{3}{*}{28} & CoI & + & + & + \\
\hline & CollI & \pm & + & \pm \\
\hline & FN & + & + & \pm \\
\hline \multirow{3}{*}{32} & CoI & + & ++ & + \\
\hline & CollI & + & + & + \\
\hline & FN & ++ & + & + \\
\hline \multirow{3}{*}{ Ad } & CoI & + & \pm & + \\
\hline & CollI & + & ++ & ++ \\
\hline & $\mathrm{FN}$ & + & ++ & ++ \\
\hline
\end{tabular}

Abbreviation: \pm , very weak reaction; + , moderate reaction; ++ , strong reaction; -, no reaction; Ant., anterior band; Int., intermediate band; Post, posterior band; Col, collagen type I; CoIII, collagen type III; FN, fibronectin.

parallel collagen bundles (Figs. 5, 6). In adults in the $40 \mathrm{~s}$, dense collagen bundles with numerous fibers arranged in a complex fashion were found in a posterior band. The fibers in these bundles were arranged anteroposteriorly, transversally, and obliquely in adults aged $40 \mathrm{~s}$. Large network structures of collagen bundles were found in the posterior band of the disk in adults aged $80 \mathrm{~s}$ to $90 \mathrm{~s}$. These network structures surrounded transversely oriented collagen bundles (Fig. 7).

\section{Discussion}

Rees (1954) and Thilander (1964) reported that the TMJ disk was a tendonous bundle that was part of the lateral pterygoid muscle. The disk is composed of anteroposteriorly, laterolaterally and obliquely arranged collagen fibers. In our results, these arranged collagen fibers were only found in the in adults aged $40 \mathrm{~s}$. Silva (1969) displayed the different structures between the surface and the inner layer of the disk. Minarelli and Liberti (1997) showed the specific structure and arrangement of collagen fibers in three bands (anterior, middle, and posterior) in the embryo, infant, and adult stages. Our results also showed the different arrangements of the collagen bundles in each band; the arrangement in the posterior band gradually became more complex during development. Changes in these specific structural arrangements may reflect the functional changes in feeding behavior that occur from suckling to mastication during development of the TMJ. The structure of the disk reflects the strong tension and pressure during mastication. In our results, numerous collagen bundles oriented anteroposteriorly and laterolaterally were always found in the middle layer of the disk from 20 weeks' gestation. In the middle layerthese bundles became compacted, dense and large during development. This structure may be displayed a tension from opening a jaw. In the posterior band, the complex arrangement of the collagen fibers differed between two groups of adults (those in their $40 \mathrm{~s}$ vs. those aged 80 to 90 years). This different structure reflects the degeneration of collagen fibers caused by years of continuous pressure and tension on the jaw. Therefore, the structure of the disk was influenced by two elements: changes in function between suckling and mastication, and degeneration or adoption of collagen fibers with age from many years of jaw movement. Rowe (1981) suggested structures of the collagen bundles changed by the movement of the lateral pterygoid muscle.

Rees (1954) reported that the disk moves from a posterior position to an anterior one, and the position of contact by the mandibular condyle changes from the posterior band to the anterior band when the jaw opens. When the jaw is opened, strong tension occurs in the bilaminar zone of the disk, which has an elastic structure that helps it to meet this demand. Griffin and Sharpe (1962) reported that elastic fibers were found mainly in the connective tissue of the posterior band.

The elastic fibers give the disk mobility under the strong tension of jaw opening. The disk changes in functional disorders of the TMJ (Öberg et al., 1971). In our study, the collagen bundles formed large network structures in the posterior band of the disk in adult aged 80 to 90 . Endomysial collagen fibrils are found in the skeletal muscle (Schmalbrauch, 1974; Ohtani et al., 1988; Fawcett, 1994), and they play a specific role in movement under stress from tightening and slackening. These morphological features of the human disk reflect the stress it is subjected to from the characteristic jaw movements associated with tooth loss, reduced dentition, and mastication. These features were found from 24 weeks' gestation, but at this young age they occurred only in the inner layer of the disk. This 
finding may be a peculiarity of the developmental stage of the disk, and it may reflect the functional difference between mastication in adults and sucking of embryonic stages. In embryonic stages, jaw movement needs to incorporate sponge-like cushioning against closing pressure.

Minarelli and Liberti (1997) observed that the human disk was composed of collagen types I and III. In the disk, collagen type I acts mainly as a structural protein. The mass and distribution of collagen type I are related to the functional properties of the disk. In our observation, the concentration of collagen type I was high in the intermediate band of the disk, compared with the anterior and posterior bands, at 32 weeks' gestation. In contrast, concentration of collagen type III was detected in the anterior and posterior bands. The differences in distribution of components of the extracellular matrix indicate specific patterns of jaw movement in the adult and fetus. Collagen type III is found mainly in soft tissues attached to bone (Keene et al., 1991), and particularly in the periosteum of intramembranous bone (Carter et al., 1991). In general, collagen type III is replaced by collagen type I at a late embryonic stage (Reddi et al., 1977; Steinmann and Reddi, 1980). We found that different concentrations of collagen type III was found from 28 weeks' gestation to 32 weeks' gestation. The concentration of fibronectin increased from 20 weeks' gestation. These differences in extracellular matrix profiles during development might reflect the differences in function as mastication or sucking.

Thilander (1964) reported an increase in the complexity arrangement of collagen fiber coinciding with a decrease in neovascularization during development. In our observation, active blood vessel development was found at 28 weeks' gestation, and large collagen bundles were found in adulthood. Wright et al. (1974) found tightness of the posterior temporal attachment of the lateral ligament and the posterior attachment of the lateral pterygoid muscle at the posterior band of the human TMJ in the postnatal stage. In adulthood, the complex and tight collagen fibers of the disk serve as strong attachments to the temporal bone and lateral pterygoid muscle. Elastic fibers are not found at 13 to 17.5 weeks' gestation (Wong et al., 1985) but occur in childhood and decrease in number with age (Thilander, 1964). We did not find elastic fibers between 12 and 32 weeks' gestation: they appeared at 40 years and 80 to 90 years. The elastic fibers may not decrease in number with age; instead, only their mass may decrease.

\section{References}

1) Akerman S, Kopp S and Rohlin M. Histological changes in temporomandibular joints from elderly individuals. Acta Odontol Scand 1986; 44:231-239.

2) Ali AM, Sharawy M, O'Dell NL and Al-Behery G. Morphological alterations in the elastic fibers of the rabbit craniomandibular joint following experimentally induced anterior disk displacement. Acta Anat 1993; 147:159-167.

3) Carter DH, Sloan $P$ and Aaron JE. Immunolocalization of collagen types I and III, tenascin, and fibronectin in intramembranous bone. J Histochem Cytochem 1991; 39:599606.

4) Fawcett DW. Striated muscle. In: Fawcett DW and Raviola E (eds): Textbook of Histology, pp. 266-267, Chapman \& Hall, New York, 1994.

5) Griffin CJ and Sharpe CJ. Distribution of elastic tissue in the human temporomandibular meniscus especially in respect to ügcompressionühareas. Aust Dent J 1962; 7:72-78.

6) Isberg AM and Isacsson G. Tissue reactions of the temporomandibular joint following retrusive guidance of the mandible. J Craniomandibular Practice 1986; 2:144-148.

7) Jonsson G, Eckerdal $O$ and Isberg $A$. Thickness of the articular soft tissue of the temporal Component in temporomandibular joints with and without disk displacement. Oral Surg Orat Med Orat Pathol Oral Radiol Endod 1999; 87:20-26.

8) Keene DR, Sakai LY and Burgeson RE. Human bone contains type III collagen, type VI collagen, and fibrillin: type III collagen is present on specific fibers that may mediate attachment of tendons, ligaments, and periosteum to calcified bone cortex. J Histochem Cytochem 1991; 39:5969.

9) Mills DK, Fiandaca DJ and Scapino RP. Morphologic, microscopic, and immunohistochemical investigations into the function of the primate TMJ disc. J Orofacial Pain 1994; 8:136-154.

10) Minarelli AM and Liberti EA. A microscopic survey of the human temporomandibular joint disc. J oral Rehabilitation 1997; 24:835-840.

11) Nagy NB and Daniel JC. Distribution of elastic fibres in the developing rabbit craniomandibular joint. Archs oral Biol 1991; 36:15-23.

12) Ohtani O, Ushiki T, Taguchi $T$ and Kikuta A. Collagen fibrillar networks as skeletal frameworks: a demonstration by cell-maceration/scanning electron microscope method. Arch Histol Cytol 1988; 51:249-261.

13) Öberg T, Carlsson GE and Fajers $C$. The temporomandibular joint, A morphologic study on a human autopsy material. Acta Odontol Scand 1971; 29:349-384.

14) Reddi AH, Gay R. Gay S and Miller EJ. Transitions in collagen types during matrix-induced cartilage, bone, and bone marrow formation. Proc Natl Acad Sci USA 1977; 74:5589-92.

15) Rees LA. The structure and function of the mandibular joint. Brit Dent J 1954; 96:125-133.

16) Rowe RWD. Morphology of perimysial and endomysial connective tissue in skeletal muscle. Tissue \& Cell 1981; 13:681-690.

17) Schmalbruch $\mathrm{H}$. The sarcolemma of skeletal muscle fiber as demonstrated by a replica technique. Cell Tissue Res 1974; 150:377-387.

18) Silva DG. Further ultrastructural studies on the temporomandibular joint of the guinea pig. J Ultrastruct Res 1969; 26:148-162.

19) Steinmann BU and Reddi $\mathrm{AH}$. Changes in synthesis of types-I and -III collagen during matrix-induced endochon- 
dral bone differentiation in rat. Biochem J 1980; 186:91924.

20) Stratmann U, Schaarschmidt K and Santamaria P. Morphometric investigation of condylar cartilage and dis thichkness in the human temporomandibular joint: siginificance for the definition of ostearthrotic changes. J Oral Pathol Med 1996; 25:200-5.

21) Streeter GL. Developmental horizons in human embryos, description of age groups XV, XVI, XVII, and XVIII. Contrib Embryol 1948; 211:135-203.

22) Streeter GL. Developmental horizons in human embryos, description of age group XII, 13 to 20 somites and age group XII, 21 to 29 somites. Contrib Embryol 1942; 197:213-245

23) Streeter GL. Developmental horizons in human embryos, description of age groups XIX, XX, XXI, XXII, and XXIII. Contrib Embryol 1951; 230:167-196.

24) Streeter GL. Weight, sitting height, head size, foot length, and menstrual age of the human embryos. Contrib Embryol 1920; $55: 146-169$.

25) Thilander $B$. The structure of collagen of the temporomandibular disc in man. Acta Odont Scand 1964; 22:135-149.

26) Wong GB, Weinberg S, Symington JM and Symington JM. Morphology of the developing articular disc of the human temporomandibular joint. J Oral Maxillofac Surg 1985; 43:565-569.

27) Wright DM and Moffet JR. The postnatal development of the human temporomandibular joint. Am J Anat 1974; 141:235-250.

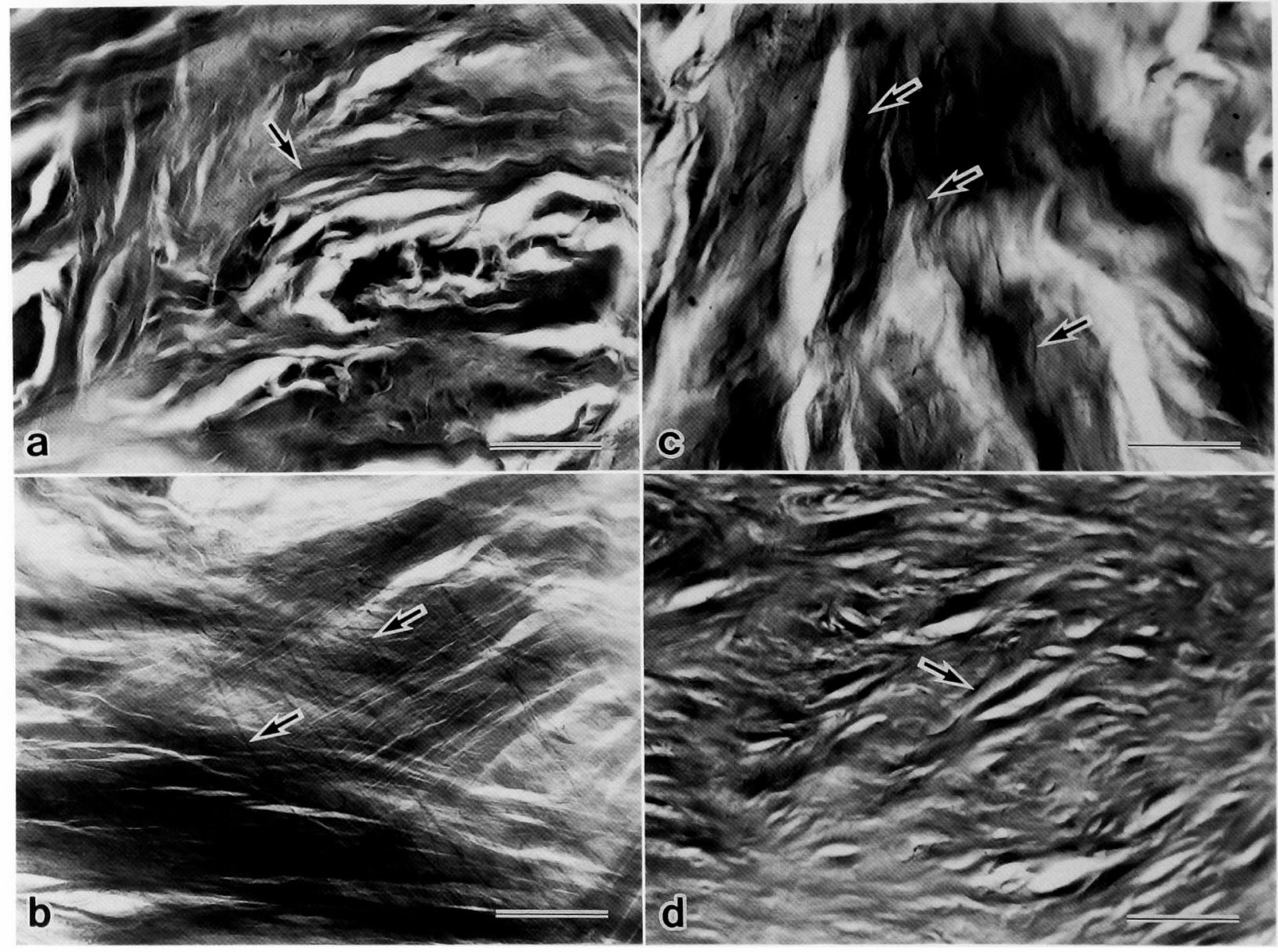

\section{Explanation of Figures}

\section{Plate I}

Fig. 1. A large magnification of sagittal-section of the chordae with Elastic van Gieson staining.

a) A few elastic fibers (Efs, arrow) were found around collagen bundles in the anterior band in the 44 years old.

b) Numerous Efs (arrows) were found in the posterior band in the 44 years old.

c) A few EFs (arrows) were concentrated in large bundle of the anterior band in the 88 years old.

d) A few Efs (arrow) were found in the posterior band in the 88 years old. (bars $=50 \mu \mathrm{m}$; a to d, same magnification). 
Plate II
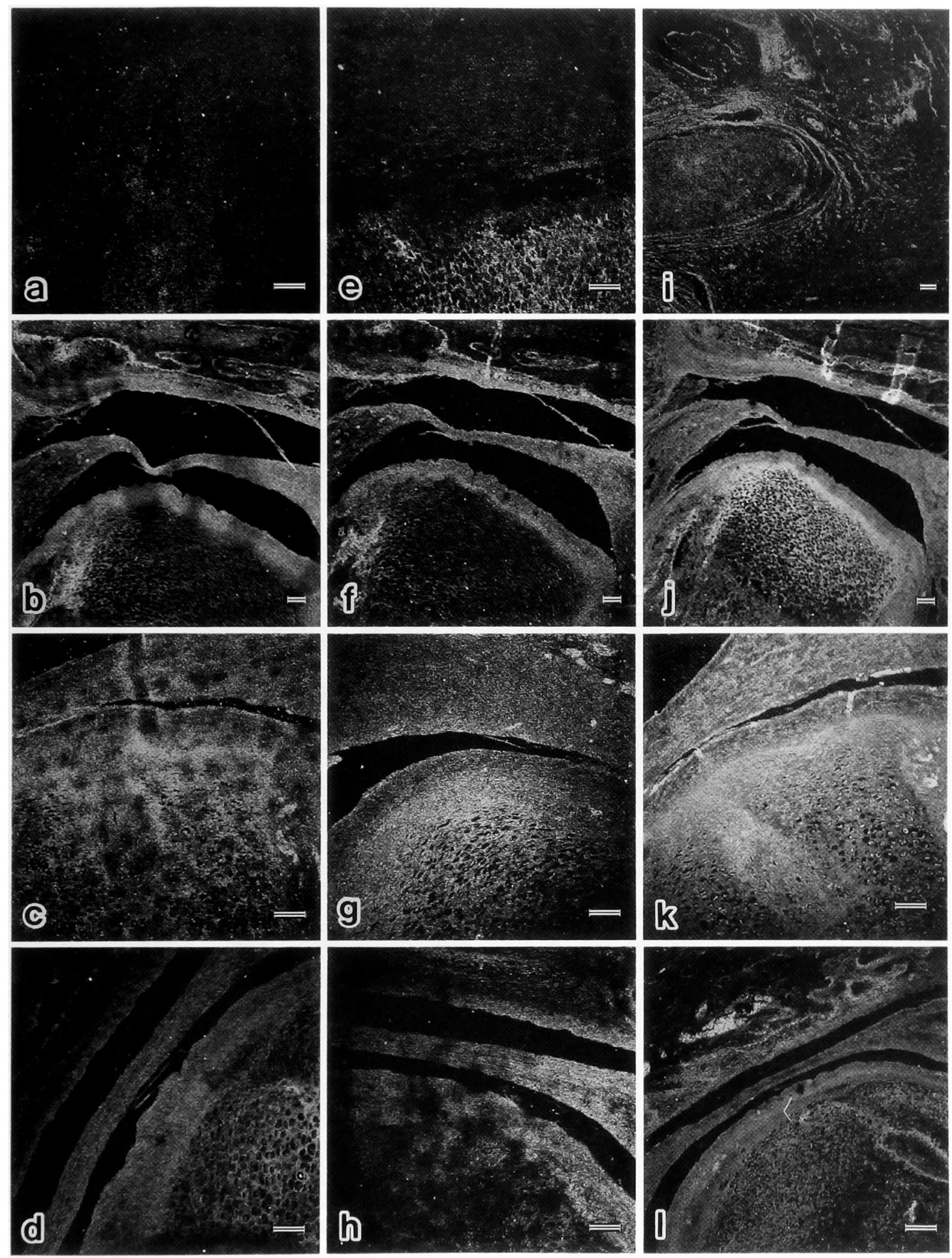

\section{Plate II}

Fig. 2. Immunohistochemical staining of the sagittal sections of the TMJ from 12 weeks' to 24 weeks' gestation. a) Collagen type I (CoI) of 12 weeks' gestation. b) Col of 16 weeks' gestation. c) The CoI of 20 weeks' gestation. d) CoI of 24 weeks' gestation. e) Collagen type III (CoIII) of 12 weeks' gestation. f) CoIII of 16 weeks' gestation. g) CoIII of 20 weeks' gestation. h) The CollI of 24 weeks' gestation. i) Fibronectin (FN) of 12 weeks' gestation. j) FN of 16 weeks' gestation. k) FN of 20 weeks' gestation. 1) FN of 24 weeks' gestation. (bars $=100 \mu \mathrm{m}$; a to l, same magnification). 



\section{Plate III}

Fig. 3. Immunohistochemical staining of the sagittal sections of the TMJ from 28 weeks' to 32 weeks' gestations. a) Collagen type I (CoI) of 28 weeks' gestation. b) Large magnification of fig. 6a. c) CoI of 32 weeks gestation. d) Large magnification of fig. 6 b. e) Collagen type III (CoIII) of 28 weeks gestation. f) Large magnification of fig. $6 \mathrm{e} . \mathrm{g}$ ) CoIII of 32 weeks gestation. h) Large magnification of fig. 6a. i) Fibronectin (FN) of 28 weeks gestation. j) Large magnification of fig. 6i. k) FN of 32 weeks gestation.

1) Large magnification of fig. $6 \mathrm{k}$. (bars $=100 \mu \mathrm{m}$; a to 1 , same magnification). 
Plate IV
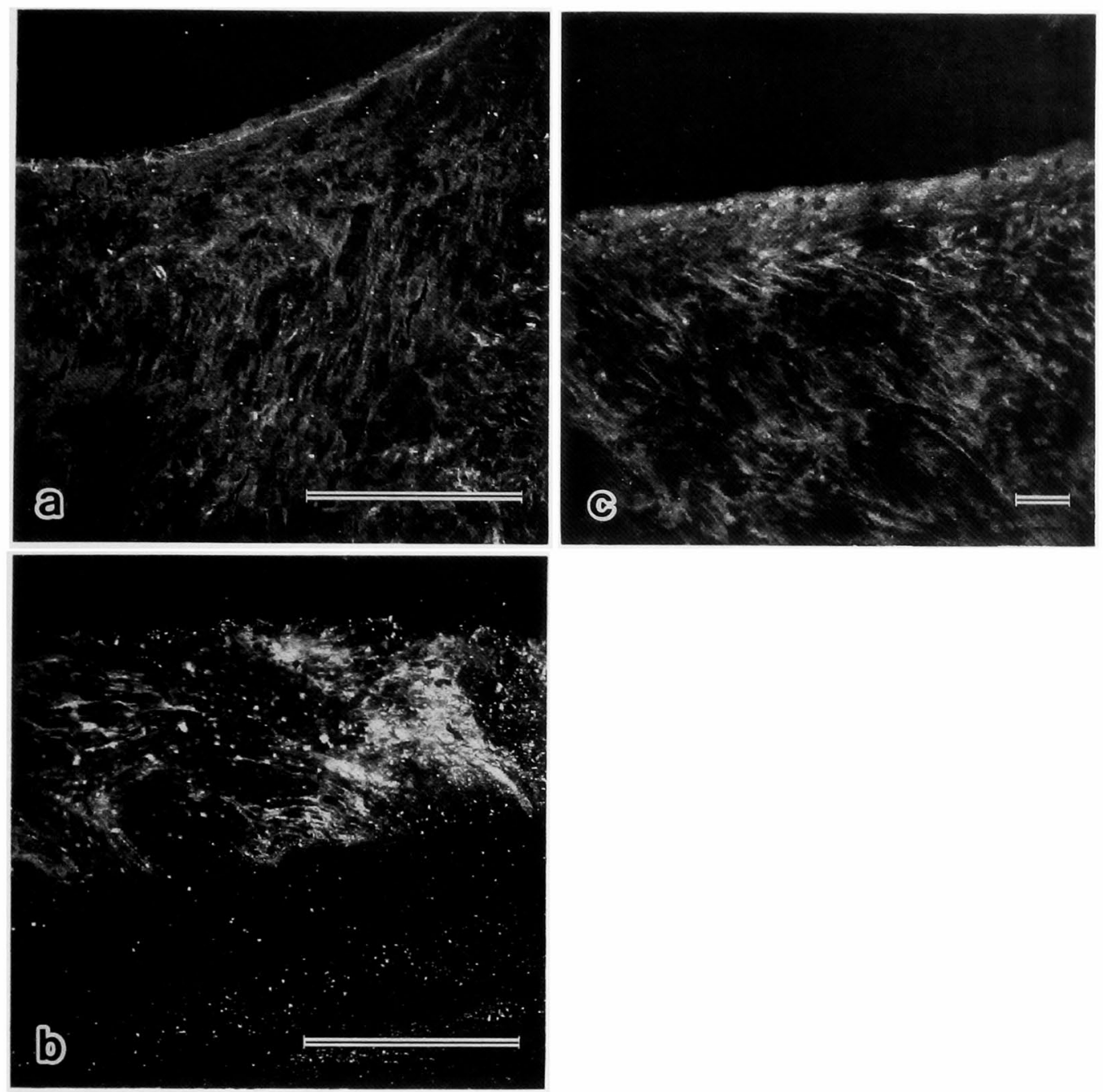

\section{Plate IV}

Fig. 4. Immunohistochemical staining of sagittal sections of the TMJ in adult aged $80 \mathrm{~s}$ to $90 \mathrm{~s}$. a) Collagen type $\mathrm{I}$ (CoI) of aged 80 in the intermediate band. b) Collagen type III (CoIII) of aged $80 \mathrm{~s}$ in the intermediate band. c) The fibronectin (FN) of aged $80 \mathrm{~s}$ in the intermediate band. d) CoI of age $90 \mathrm{~s}$ in the posterior band. e) CoIII of age $90 \mathrm{~s}$ in the posterior band. f) FN age $90 \mathrm{~s}$ in the posterior band. (bars $=100 \mu \mathrm{m}$; a to f, same magnification).

\section{Plate V}

Fig. 5. Scanning electron microscopy of the TMJ from 12 weeks' gestation to 24 weeks' gestation. IBD, intermediate band of the disk; PBD, posterior band of the disk.

a) IBD in 12 weeks gestation (bar $=10 \mu \mathrm{m})$.

b) PBD in 12 weeks gestation (bar $=5 \mu \mathrm{m}$ ).

c) IBD in 16 weeks gestation (bar $=20 \mu \mathrm{m}$ ).

d) PBD in 16 weeks gestation (bar $=20 \mu \mathrm{m}$ ).

e) IBD in 20 weeks gestation ( $\mathrm{bar}=50 \mu \mathrm{m})$.

f) PBD in 20 weeks gestation (bar $=20 \mu \mathrm{m}$ ).

g) IBD in 24 weeks gestation (bar $=10 \mu \mathrm{m})$.

h) PBD in 24 weeks gestation ( $b a r=20 \mu \mathrm{m}$ ). 


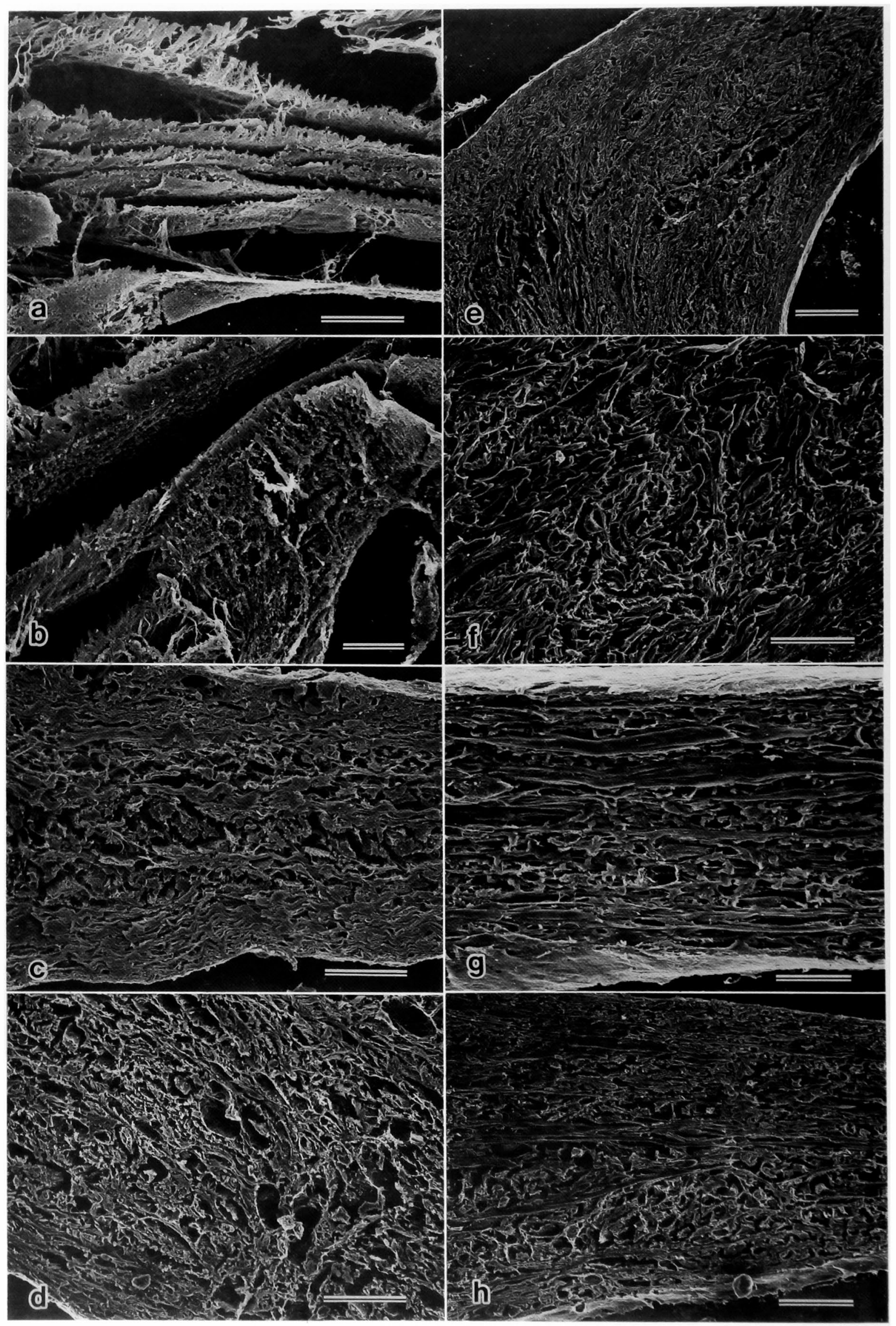


Plate VI

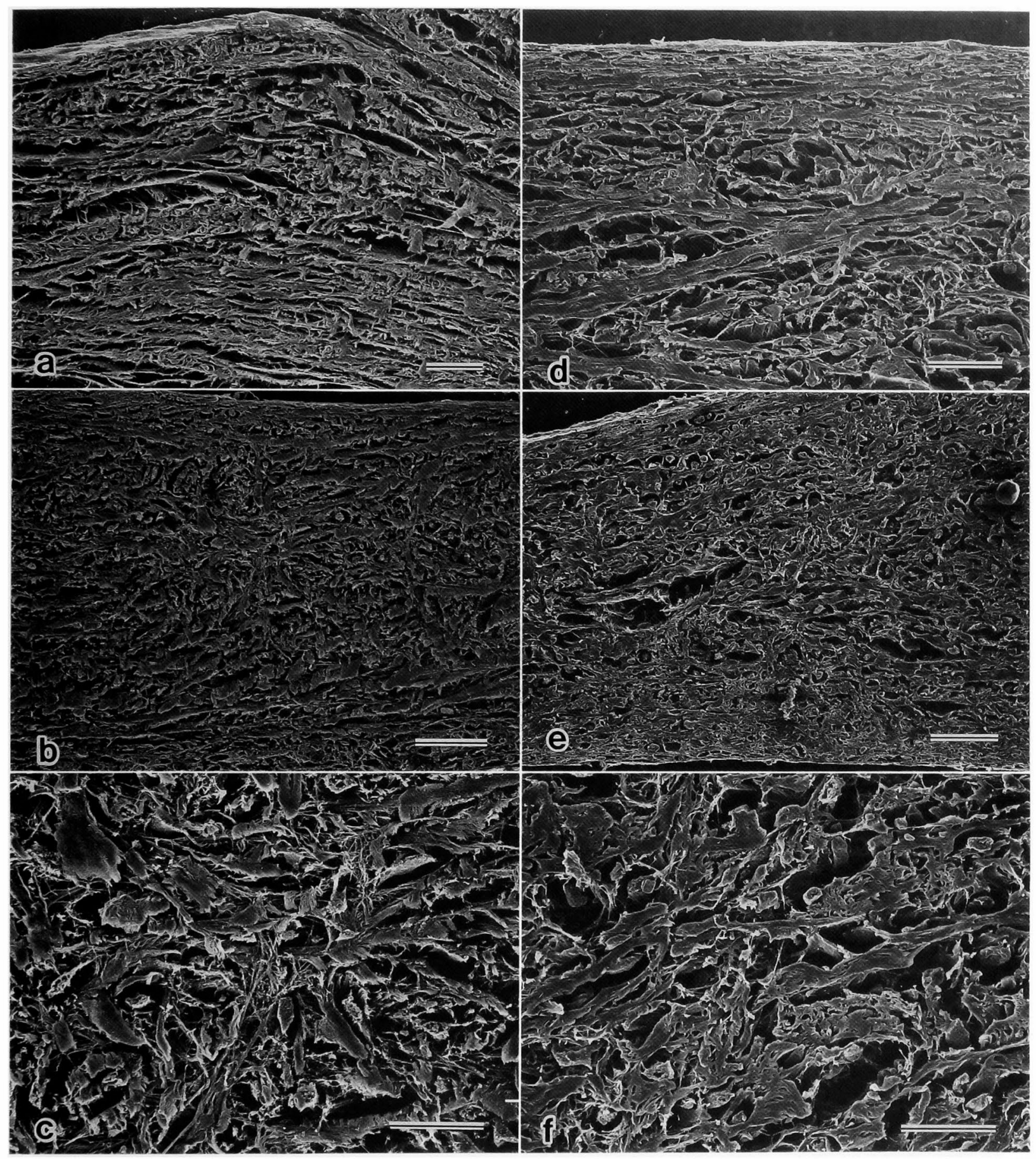

\section{Plate VI}

Fig. 6. Scanning electron microscopy (SEM) of the TMJ from 28 weeks' gestation to 32 weeks' gestation. IBD, intermediate band of the disk; PBD, posterior band of the disk.

a) IBD in 28 weeks gestation (bar $=50 \mu \mathrm{m}$ ).

b) PBD in 28 weeks gestation (bar $=30 \mu \mathrm{m})$.

c) Large magnification of Fig. $6 \mathrm{~b}(\mathrm{bar}=20 \mu \mathrm{m})$.

d) PBD in 32 weeks gestation (bar $=30 \mu \mathrm{m}$ ).

e) IBD in 32 weeks gestation ( $\mathrm{bar}=40 \mu \mathrm{m})$.

f) Large magnification of Fig. $6 \mathrm{e}(\mathrm{bar}=20 \mu \mathrm{m})$. 

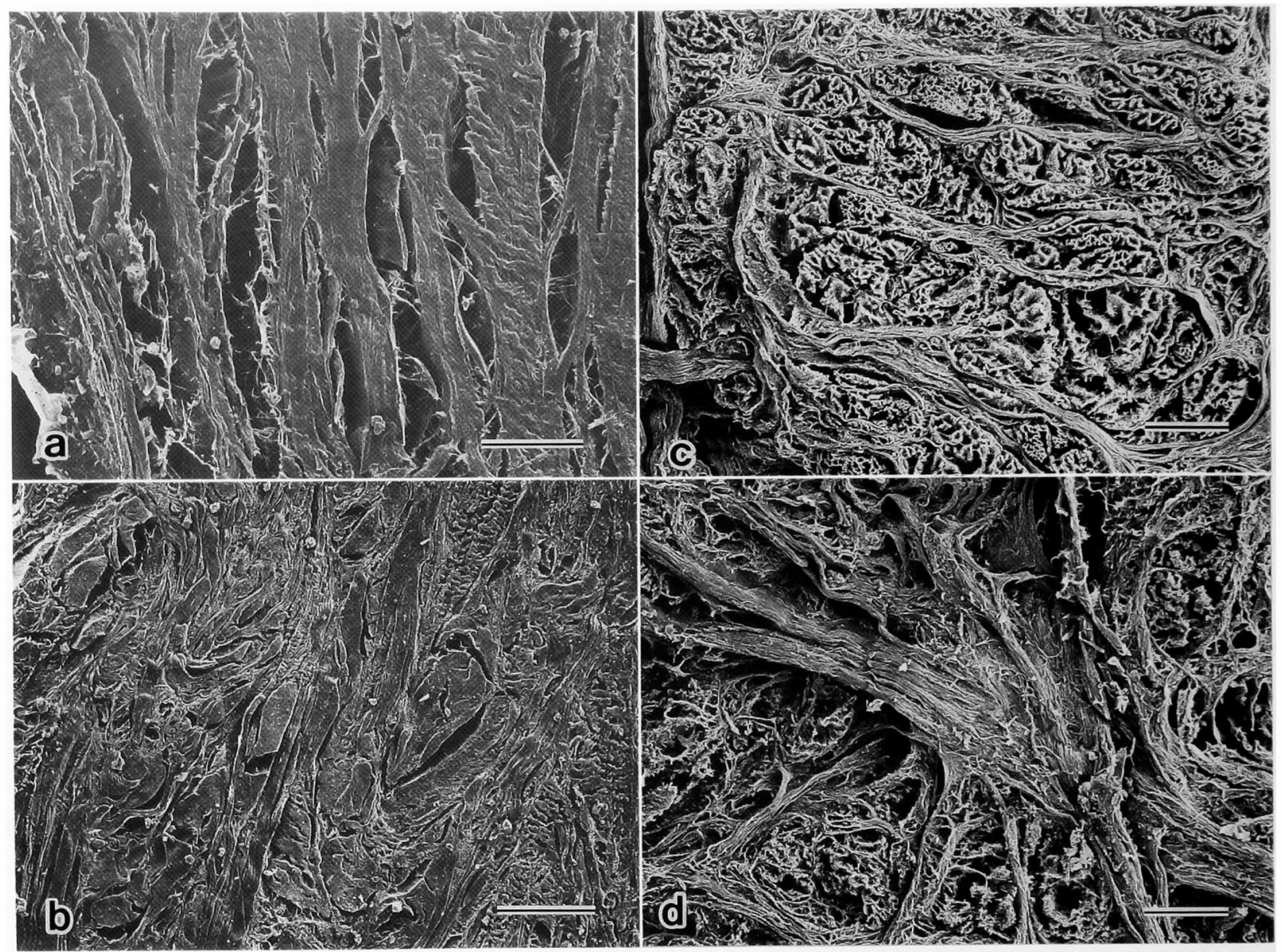

\section{Plate VII}

Fig. 7. Scanning electron microscopy (SEM) of the TMJ both age of 44 and age of 90 . IBD, intermediate band of the disk; PBD, posterior band of the disk.

a) IBD in age of $44(\mathrm{bar}=40 \mu \mathrm{m})$.

b) PBD in age of $44(\mathrm{bar}=40 \mu \mathrm{m})$.

c) IBD in age of $90(\mathrm{bar}=40 \mu \mathrm{m})$.

d) PBD in age of $99(\mathrm{bar}=40 \mu \mathrm{m})$ (bars $=40 \mu \mathrm{m}$; a to d, same magnification). 